\title{
Successive crossover from ordinary Born scattering to multiple scattering to localization - A delay time analysis in electronically random systems
}

\author{
Sandeep K. Joshi [a] and A. M. Jayannavar [b] \\ * Institute of Physics, Sachivalaya Marg, Bhubaneswar 751 005, India
}

\begin{abstract}
We have studied the reflection delay time distribution from a one-dimensional electronically random system of finite length. We show that the average delay time is a non-monotonic function of the strength of the disorder and exhibits three qualitatively different regimes. In the weak disorder limit the scattering is dominated by the ordinary Born scattering. For the intermediate strengths of disorder a well defined regime of multiple scattering emerges followed by the localization domain.
\end{abstract}

Key words: A. disordered systems, D. electronic transport, D. quantum localization

\section{INTRODUCTION}

Wigner delay time, the energy derivative of scattering phase shift, is considered to be related to the time spent by the particle in the region of interaction [1,2]. Since its inception in 1955 the Wigner delay time has been a quantity of profound interest from fundamental as well as technological point of view [3-6]. The delay time statistics is intimately connected with the dynamic admittance of microstructures [3]. Although the phase delay time alone is not sufficient to calculate capacitance of extended mesoscopic systems, a direct relation between the capacitance and the phase time does exist for systems which can be treated essentially as zero dimensional [6] (eg. a cavity or a quantum dot). The wave packet incident on the surface of a sample is backscattered after some delay. In random systems this results in a low temperature $1 / f$ type noise for the fluctuating surface currents due to non-cancellation of the instantaneous currents at the surface [7]. For a three dimensional system of arbitrary shape with an arbitrary number of incoming channels the density of states is proportional to the sum of dwell times for all incoming channels [8]. In case of a one dimensional disordered sample connected to an infinite lead the dwell time is the same as the Wigner delay time. This delay time is directly related to the density of states.

In the single channel case the distribution of the delay time for a disordered semi-infinite sample was obtained earlier by using the invariant imbedding approach [7]. The stationary distribution $P_{s}(\tau)$ for the dimensionless delay time $\tau$ is 
given by

$$
P_{s}(\tau)=\frac{\lambda e^{\lambda t a n^{-1}} \tau}{\left(e^{\lambda \pi / 2}-1\right)\left(1+\tau^{2}\right)},
$$

where $\lambda$ is proportional to the disorder induced localization length and the most probable value of $\tau$ occurs at $\tau_{m p}=\lambda / 2$. The long time tail of the above distribution scales as $1 / \tau^{2}$. The average value of $\tau$ is logarithmically divergent indicating the possibility of the particle traversing the infinite sample before being totally reflected, due to the resonances $[9,10]$. If the disordered region is semi-infinite or if it is terminated at one end by an infinite wall, the reflection coefficient will be unity, and the complex reflection amplitude will have the form $R=e^{i \theta(E)}$.

A recent study based on analytical work found the delay time distribution in the one-channel case to be universal (especially the long time tail is independent of the nature of disorder) [11] within random phase approximation (RPA). Soon after the universality of tail distribution beyond RPA was established numerically [12]. The universality of stationary distribution of delay time was further supported by another analytical work where the random potential is of a different kind [13]. So far in this field the localization has been studied extensively. Recently Texier et. al. [9] have studied the statistics of delay time distribution for ballistic case for which sample size is much smaller than the localization length. In the present paper we analyse the statistical properties of delay time and study the emergence of three qualitatively different regimes of transport or scattering in a finite one-dimensional random system. These different domains are dominated by ordinary Born scattering, multiple scattering and localization respectively. The crossover from one regime to another as the disorder strength is varied is studied.

\section{NUMERICAL TECHNIQUE}

The following Hamiltonian, written in a tightbinding, one-band model is used to describe the motion of a quasiparticle on a random lattice:

$H=\sum \epsilon_{n}|n\rangle\langle n|+V(|n\rangle\langle n+1|+| n\rangle\langle n-1|),(2)$

where $|n\rangle, \epsilon_{n}$ and $V$ denote the non-degenerate Wannier orbital at site $n$, the site energy at the site $n$ and the hopping matrix element connecting nearest neighbors separated by a unit lattice spacing respectively. We consider site diagonal disorder where the site energies $\epsilon_{n}$ are assumed uncorrelated random variables distributed uniformly over $-W / 2$ to $W / 2\left(P\left(\epsilon_{n}\right)=1 / W\right)$. The $L$ site $(n=1$ to $L)$ disordered 1D sample is connected at one end to a perfect infinite lattice having all site energies zero. The well-known transfer-matrix method $[14,15]$ is used to calculate the reflection amplitude $r(E)=|r| e^{-i \theta(E)}$ and its phase $\theta(E)$ at two values of incident energy $E=E_{0} \pm \delta E$. The delay time is then calculated using the definition $\tau=\hbar d \theta / d E$.

Throughout our following discussion we consider the delay time $\tau$ in a dimensionless form by multiplying them with $V$ and we set $\hbar=1$. For all the results discussed below we have used $E_{0}=0$ and $\delta E=10^{-8}$. We have confirmed the stability of our results with respect to choice of $\delta E$ within the range $10^{-6}<\delta E<10^{-9}$. For the calculation of various distributions and averages we have used atleast $10^{6}$ realizations of the disordered sample.

\section{Results and discussion}

In Fig. 1 we show the plot of average value of delay time $\langle\tau\rangle$ versus the disorder strength for a sample of length $L=50$. For very small values of $W$ such that localization length $\xi \gg L\left(\xi=96 / W^{2}[15]\right)$, we see that the $\langle\tau\rangle$ decreases with increasing $W$. This is the ballistic regime. Since the disorder is very weak i.e., $\xi \gg L$ Born scattering dominates. 
Due to this the waves undergo mostly single scattering events. The $\langle\tau\rangle$ continues to fall upto a certain strength of disorder depending upon the length of the sample. At this strength of disorder $\xi \sim 20 L$. We have verified this for samples of different lengths. Increasing $W$ beyond this value we observe that $\langle\tau\rangle$ starts increasing with disorder, i.e., the disorder becomes strong enough such that multiple scattering events start dominating. This leads to particles (or waves) spending more time in the sample before getting reflected. Consequently, the reflection delay time increases with increasing disorder. This trend continues until the disorder becomes strong enough such that $\xi \sim L$. On increasing $W$ beyond this, a crossover to localization occurs. In this regime the most probable distance traversed by the particles is twice the localization length. The localization length decreases with disorder strength as $96 / W^{2}$. Therefore, the most probable value of the reflection delay time, the time required to travel to-andfro distance of $\xi$, also decreases and so does $\langle\tau\rangle$. However, from the inset shown in the figure we see that the root mean squared relative variance $\sigma_{r m s}=\sqrt{\left\langle\tau^{2}\right\rangle-\langle\tau\rangle^{2}} /\langle\tau\rangle$ in this regime is much larger than unity. This indicates that the fluctuations in $\tau$ in this regime are very large and hence $\tau$ is a non-self-averaging quantity. Thus, from the behaviour of average reflection delay time $\langle\tau\rangle$ we are able to identify three qualitatively different regimes of wave propagation in one-dimensional disordered medium.

In Fig. 2 is shown the distribution $P(\tau)$ of reflection delay time $\tau$ from a sample of fixed length $L=50$ for three values of disorder strength $W$ in the ballistic regime. For $W=0$ the distribution is a delta peak at a value of $\tau$ equal to the round trip delay time $\tau_{0}$. For the weak disorder case the most probable value of $\tau$ is the round trip delay time $\tau_{0}$. From the figure we observe that the distributions are asymmetric about the most probable value $\tau_{0}$. The distribution has higher weight for values $\tau<\tau_{0}$ and lower weight for values of $\tau>\tau_{0}$. The asymmetry is confirmed by looking at the third moment of $\tau$ which is $\chi_{s}=\left\langle(\tau-\langle\tau\rangle)^{3}\right\rangle$ shown in the inset of Fig. 2. The variation of $\chi_{s}$ with $W$ sheds some light on the behaviour of $\langle\tau\rangle$ with $W$ in the ballistic regime. In this regime, as mentioned earlier, disorder is weak and much of the behaviour is dominated by single scattering events which occur anywhere in the sample before reaching endpoint. As the disorder strength is increased the probability of scattering at individual sites also increases. Thus, the probability of particle encountering a scattering near the surface itself increases with increasing disorder strength. This enhances the weight for small $\tau$ in $P(\tau)$. Although, the multiple scattering events contribute to the distribution for $\tau>\tau_{0}$, the change in their contribution with increasing disorder is not much compared to that of single scattering events.

The distributions of delay time in the multiple scattering regime and the localized regime are shown in Fig. 3. For the distribution in the multiple scattering regime we notice that the most probable delay time $\tau_{m p}$ is much lower than the round trip delay time $\tau_{0}$. The distribution is broader and has a tail. The appearance of a broad distribution and a long time tail indicates that in this regime the multiple scattering events are indeed dominating. In fact, it is due to the appearance of a tail that the $\langle\tau\rangle$ increases with increasing disorder strength $W$ in spite of the reduction in $\tau_{m p}$. For $W>\sqrt{2}(\xi<L)$ we crossover to the localized regime. The corresponding distributions (for $W=1.4$ and $W=2.4$ ) are shown in the inset of Fig. 3. The $\tau_{m p}$ in this case is proportional to the typical length traversed by the particles that is the localization length $\xi$ [7]. Since $\xi$ decreases with increasing $W$ so does $\tau_{m p}$. The distribution shows a long-time-tail due to the presence of resonances. For these resonant realizations the particle explores the whole of the sample before being totally reflected. Also, for this case one can notice that the distribution becomes narrow and weight at the tails becomes smaller with increasing disorder in contrast with the behaviour observed for the tails in the multiple scattering domain.

In the ballistic regime the transport is dominated by few scattering events and since the disorder is weak the phase changes associated with these are 
small. Consequently, the distribution of phase of the reflected wave shows (Fig. 4) a doubly peaked structure in this regime. The peaks occur at 0 and $2 \pi$. In the multiple scattering regime the multiple scattering events provide ample opportunity for the phase to get randomized. This is reflected in the fact that the distribution shown in Fig. 4 for $W=0.7$ (multiple scattering) is fairly uniform over the range 0 to $2 \pi$. For $W=8.0$ we are in the strong localization regime and once again the double peak structure emerges in the phase distribution. In this case, though the double peak structure arises because $\xi \ll L$ i.e., the scattering is so strong that most of the particles are reflected back from near the surface thus getting scattered only from a few sites. In the limit of $W \rightarrow \infty$ the peaks shift towards $\pi / 2$ and $3 \pi / 2$.

In Fig. 5 we plot the average value of time delay $\langle\tau\rangle$ as a function of length $L$ of the sample. As predicted by Texier et. al. [9] we see that the dependence is linear on $L$. In the inset of the figure we plot, on log-log scale, the second cumulant of $\tau\left\langle\tau^{2}\right\rangle-\langle\tau\rangle^{2}$ as a function of the length $L$ of the sample. We find a $L^{3.56}$ dependence of the second cumulant of $\tau$ on the length of the sample in contrast to the $L^{3}$ behaviour predicted by Texier et. al. [9]. The difference, probably, can be attributed to the approximations involved in the analytical calculations or the constraint of averaging over finite number of realizations in our numerical calculations.

\section{Conclusion}

In summary, our study based on the statistics of Wigner delay time distribution, shows that three qualitatively different regimes of transport or scattering exist in disordered finite onedimensional systems. The systems exhibit a successive crossover from ballistic regime to multiple scattering regime to localization regime as the strength of disorder is varied.

\section{References}

[a] e-mail: joshi@iopb.res.in

[b] e-mail: jayan@iopb.res.in

[1] E. P. Wigner, Phys. Rev.98, (1955) 145

[2] F. T. Smith, Phys. Rev.118, (1960) 349

[3] M. Büttiker, H. Thomas and A. Prêtre, Phys. Lett. A180, (1993) 364; N. Kumar and A. M. Jayannavar, Phys. Rev. B 32, (1985) 3345

[4] V. A. Gopar, P A. Mello and M. Büttiker, Phys. Rev. Lett. 77, (1996) 3005

[5] N. Kumar and A. M. Jayannavar, Mod. Phys. Lett. B11, (1997) 53

[6] M. Büttiker, J. Phys. Condensed Matter 5, (1993) 9361

[7] A. M. Jayannavar, G. V. Vijayagovindan and N. Kumar, Z. Phys. B75, (1989) 77

[8] G. Iannaccone, Phys. Rev. B 51, (1995) 4727

[9] Christophe Texier and Alain Comtet, condmat/9812196

[10] C. J. Bolton-Heaton et. al., cond-mat/9902335

[11] Alain Comtet and Christophe Texier, J. Phys. A Math. Gen. 30, (1997) 8017

[12] S. K. Joshi and A. M. Jayannavar, Phys. Rev. B 58, (1998) 1092

[13] S. K. Joshi and A. M. Jayannavar, Solid State Commun. 106, (1998) 363

[14] See, e.g., Y. Liu and K. A. Chao, Phys. Rev. B 34, (1986) 5247; P. K. Thakur, C. Basu, A. Mookerjee and A. K. Sen, J. Phys. Condens. Matter 4, (1992) 6095

[15] E. N. Economou, Green's Functions in Quantum Physics, $2^{\text {nd }}$ ed. (Springer-Verlag, Berlin, 1983), p. 174. 


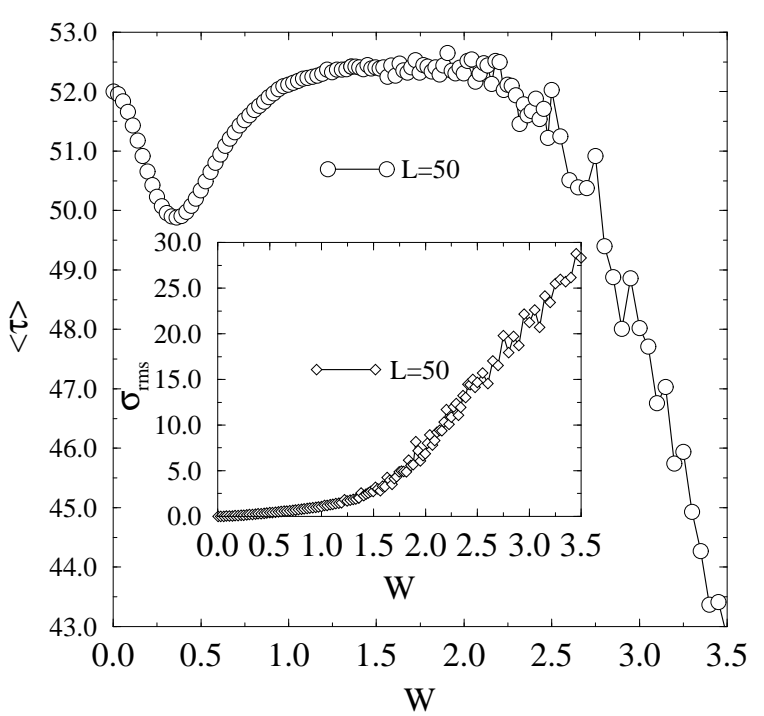

Fig. 1. Plot of $\langle\tau\rangle$ versus disorder strength $W$ for different lengths of the disordered segment. The disorder averaging is done over $10^{7}$ samples.

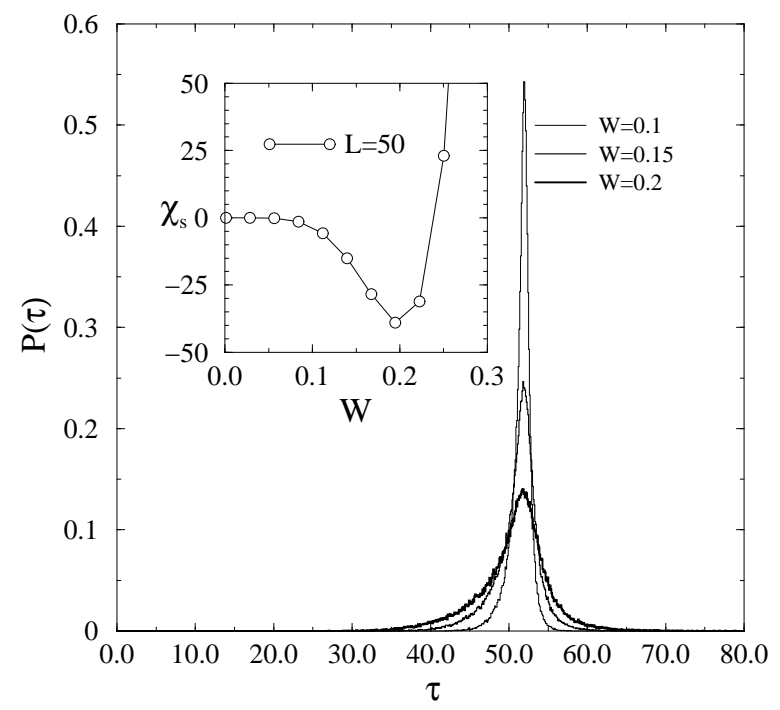

Fig. 2. Distribution of $\tau$ from a sample of length 50 for three different values of disorder strength $W$ for the ballistic regime $\xi \ll L$. The inset shows the behaviour of third moment of $\tau$ with $W$.

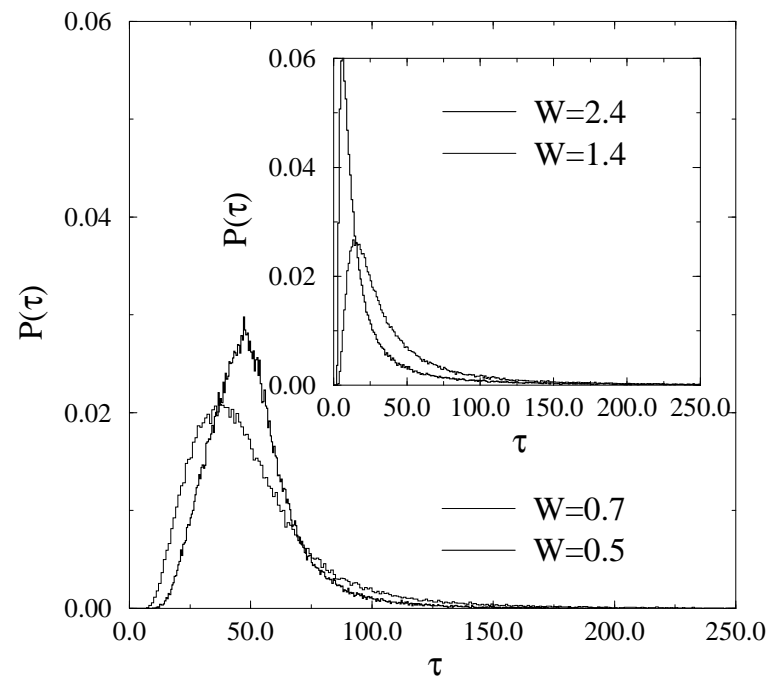

Fig. 3. Distribution of $\tau$ from a sample of length $L=50$ for different values of disorder strength $W$. For $L=50, W=0.5$ and $W=0.7$ lie in the multiple scattering regime. Inset shows the distribution of $\tau$ for $W=1.4$ and $W=2.4$ belonging to the localization regime.

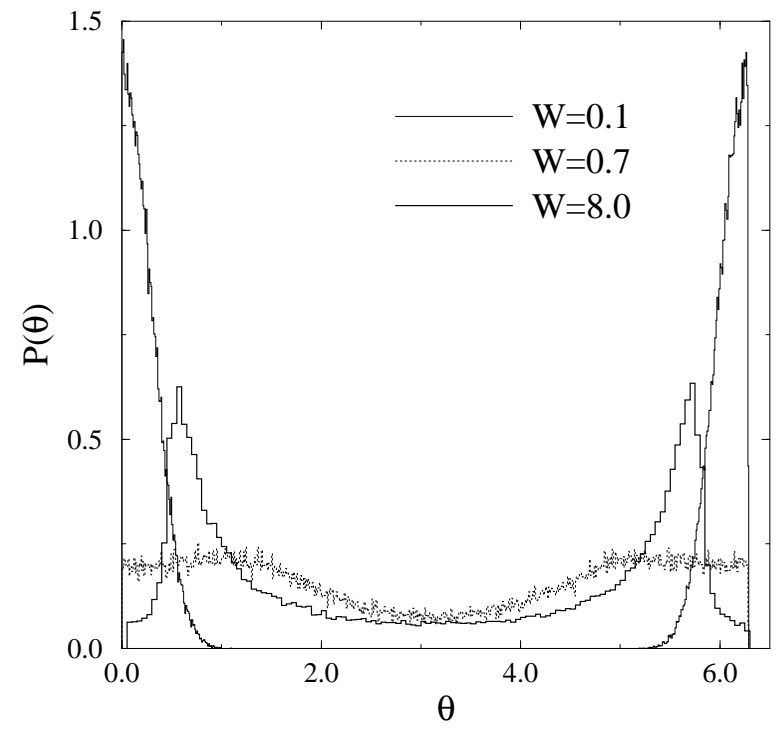

Fig. 4. Distribution of phase of reflected wave from the semi-infinite disordered sample of length $L=50$ for different values of disorder strength $W$ in the different regimes namely, ballistic $(W=0.1)$, multiple scattering $(W=0.7)$ and strong localization $(W=8.0)$. 


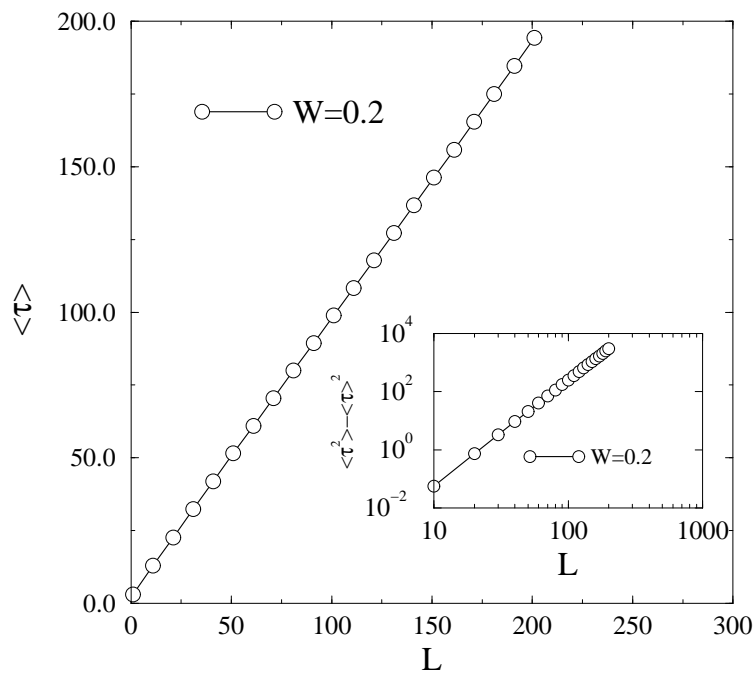

Fig. 5. Plot of Average delay time $\langle\tau\rangle$ versus length of the sample $L$ for disorder strength $W=0.2$. In the inset is the plot of second cumulant of $\tau\left(\left\langle\tau^{2}\right\rangle-\langle\tau\rangle^{2}\right)$ versus lenth of the sample $L$ for $W=0.2$. 